\title{
RESISTIVE PROCESSES IN THE PREFLARE PHASE OF ERUPTIVE FLARES
}

The Role of the Perpendicular Magnetic Fields

T. MAGARA

Univ. of Kyoto, Faculty of Science

Sakyo-ku, Kyoto 606, Japan

AND

K. SHIBATA

National Astronomical Observator

Mitaka, Tokyo 181, Japan

\begin{abstract}
In this study, we perform 2.5-dimensional MHD simulations and clarify the role of perpendicular magnetic fields (which are perpendicular to the 2D plane) in a preflare current sheet of solar flares. At the first stage, a current sheet formed within a coronal magnetic structure is filled with the perpendicular fields (force-free structure). Then this sheet begins to be dissipated through the tearing instability under a uniform resistivity. As the instability proceeds, the distribution of the perpendicular fields vary in such a way that most of $t$ hem gather around O-point (magnetic island) instead of X-point. Therefore, the magnetic pressure of these fields weaken in the vicinity of $\mathrm{X}$-point so that they no longer suppress the inflows toward this point. These flows then make the current sheet thinner and thinner, which implies that the current density around X-point becomes high enough to cause an anomalous resistivity whose value is much larger than that of the normal collisional resistivity. In this way, the transition from a uniform resistivity to a locally-enhanced one occurs, which can make the violent energy release observed in solar flares.
\end{abstract}

\section{References}

Magara, T., Shibata, K., and Yokoyama, T. (1997), ApJ, Vol.487, pp.437 Ohyama, M. and Shibata, K. (1997), PASJ, Vol.49, pp.249 


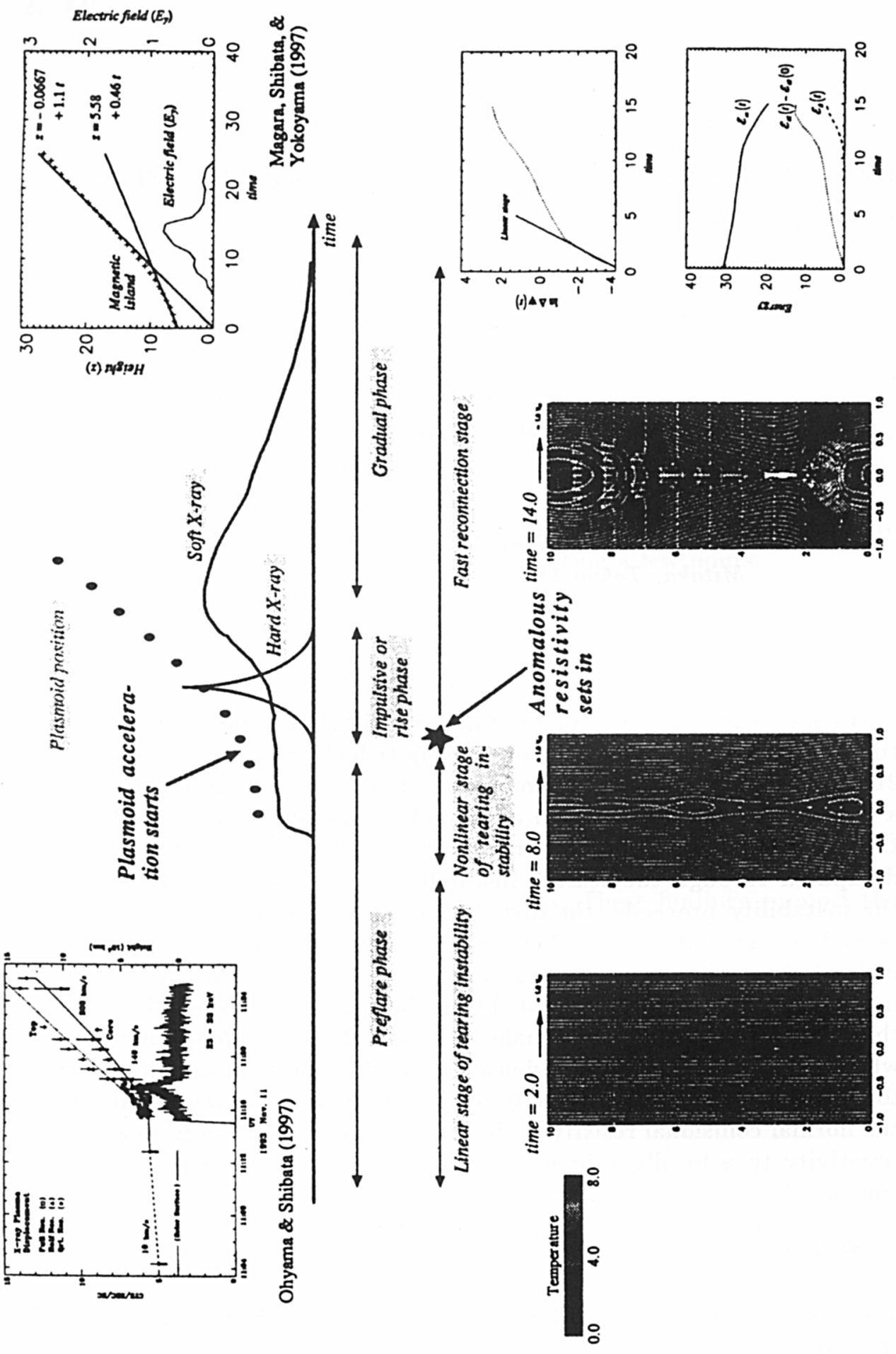

